\title{
MÉTROLOGIE D'ONDES DE CHOC À L'AIDE DE POLYMÈRES PIÉZOÉLECTRIQUES
}

\section{F. BAUER}

Institut Franco-Allemand de Recherches de Saint-Louis, 12, rue de I'Industrie, F-68301 Saint-Louis Cedex, France

Résumé : Lors de travaux précédents, il a été montré que les films polymères piézoélectriques en PVDF soumis à l'action d'une onde de choc libéraient des impuisions de courant électrique bien définies à des pressions allant jusqu'à quelques dizaines de GPa. L'intérêt de l'emploi de ces impulsions de courant pour les mesures temporelles de variations de contrainte a été clairement démontré. La présente note est un bref résumé de l'étude de la réponse piézoélectrique de jauges standards PVDF ISL-Metravib entre 0,3 et $20 \mathrm{GPa}$. Certaines déviations observées à haute pression trouvent une explication à partir d'expériences sous choc effectuées sur des jauges PVDF polarisées de façon nouvelie.

Abstract - Prior work reported that shock-compressed PVDF piezoelectric polymer films can exhibit well defined current pulse outputs to many tens of $\mathrm{GPa}$. The utility of these measured current pulses as time-resolved stress rate measures has well been demonstrated. The present report is a brief summary of the study of the piezoelectric response of standardized PVDF ISL METRAVIB gauges from 0.3 to $20 \mathrm{GPa}$. Some deviations observed at high pressure, are explained via experimentations done on new poled PVDF gauges.

\section{1 - Introduction}

Le passage d'une onde de choc dans un matériau a pour effet de le soumettre à une compression suivie d'une détente. La connaissance de cette surpression en fonction du temps constitue l'un des paramètres importants de la physique des chocs. Cette branche de la physique a beaucoup évolué au cours des quarante dernières années. Des progrès importants ont été réalisés grâce à l'augmentation du pouvoir de résolution temporelle des systèmes d'instrumentation utilisés pour étudier la réponse des matériaux soumis à l'action d'une onde de compression par choc.

Historiquement cette évolution a suivi les progrès des systèmes et capteurs mis en oeuvre : jauges chronométriques, mesures optiques en continu des phénomènes, mesures directes de la contrainte ou de la vitesse particulaire (quartz ou VISAR).

Le polyfluorure de vinylidène, PVDF, est connu pour présenter des propriétés ferroélectriques et piézoélectriques uniques en métrologie des ondes chocs. II possède la particularité de fournir une mesure directe de la variation de la contrainte en fonction du temps avec ine sensibilité que l'on peut qualifier de remarquable $[2,8,10]$.

Une jauge à choc est typiquement détruite lors de son utilisation : de ce fait, il est impossible de la calibrer, c'est-à-dire de donner d'une façon arbitraire sa réponse piézoélectrique dans un domaine étendu de pression. Si l'on ne dispose pas d'un élément piézoélectrique reproductible, il est illusoire de vouloir déterminer de façon expérimentale la réponse piézoélectrique de capteurs PVDF soumis à l'action d'une onde de choc. Le physicien, l'expérimentateur, l'ingénieur, le technicien etc., c'est- 
à-dire l'homme de I'ART souhaitant utiliser ce "capteur nouveau", doit être assuré que chaque élément PVDF préparé et polarisé aujourd'hui présentera les mêmes caractéristiques sous choc que celui polarisé demain ou dans dix ans.

Ceci est d'autant plus vrai que l'étude et la mise au point d'un capteur "nouveau" en PVDF piézoélectrique demandent des périodes d'études et de développements 'supérieures à une décennie. La nature complexe de la physique des ondes de choc et de ses effets sur les proprietés physiques pose des problèmes au niveau de la calibration du PVDF car c'est un polymère fort complexe.

Le présent travail rassemble, en résumé, l'étude de la réponse piézoélectrique sous choc des jauges PVDF. ISL fabriquées par METRAVIB dans la gamme 0,3-20 GPa. II aborde les modes de fonctionnement de ces polymères piézoélectriques. Il présente les premiers résultats obtenus avec des capteurs de laboratoire ISL en PVDF élaborés de façon différente et dont les performances apparaissent supérieures.

\section{2 - Procédé expérimental}

L'étalonnage des jauges PVDF [2, 3, 4], c'est-à-dire la détermination expérimentale de la relation entre la charge électrique libérée par la jauge et la pression de choc, nécessite l'utilisation de matériaux dont les caractéristiques sous choc sont parfaitement connues, tels que le saphir coupe $Z$, le quartz coupe $Z$, le Kel-F ou polychlorotrifluoroéthylène.

L'étalonnage des jauges est effectué à l'aide du générateur d'onde de choc calibrée mis au point à I'ISL [1, 2, 3, 4]. Ce générateur est constituté d'un canon à poudre au calibre de $15 \mathrm{~mm}$ permettant d'accélérer un projectile sous vide primaire $(0,01 \mathrm{mmHg})$ et d'induire, lors de l'impact du projectile sur une cible placée à la bouche du canon, une onde de choc plane calibrée en pression et en durée. Cet équipement a été conçu pour que l'angle entre l'impacteur et la cible soit négligeable.

L'impact symétrique de l'impacteur et de la cible crée l'onde de compression. Le capteur PVDF d'épaisseur $25 \mu \mathrm{m}$ est collé sur la surface du matériau impacté avec une colle époxy Hysol RE 2038 + Hysol HD 3404. Dans certains cas, il est isolé avec des films PFA Téflon (épaisseur 10 ou 25 microns), si les matériaux impacteurs el impactés sont conducteurs.

La précision du calcul de la pression de choc est celle de la mesure de la vitesse du projectile, soit $0,5 \%$.

Dans la configuration (projectile et cible en matériau identique), la contrainte initiale induite est celle générée par l'impacteur dans le capteur PVDF.

L'onde de choc se réfléchit entre le projectile et la cible jusqu'à ce que la contrainte s'équilibre à une valeur égale à celle atteinte pour les matériaux standards (quartz coupe Z, Kel-F, saphir coupe $Z$, carbure de tungstène) utilisés pour l'impacteur et la cible $[10,12]$.

Le signal électrique délivré par le PVDF est enregistré en mode "courant électrique". Les intensités électriques mesurées variant entre 1 et $40 \mathrm{~A}$, on utilise des sondes de courant coaxiales purement résistives ou "current viewing resistor" de résistance nominale comprise entre 0,01 et $1 \Omega$. Les câbles de mesure utilisés sont en mousse diélectrique, donc à très faibles pertes. Les temps de montée du courant $\mathrm{I}(\mathrm{t})$ sont typiquement de 2 à 6 ns. Les signaux en courant sont enregistrés au 
moyen d'enregistreurs de transitoires LeCroy $6880 \mathrm{~B}$ (bande passante $400 \mathrm{MHz}$ ) et Tektronix DSA 602 à bande passante égale à $1 \mathrm{GHz}$. Le courant est proportionnel à la différence des contraintes entre les électrodes du capteur PVDF. Le courant émis par la jauge est fonction de la variation temporelle de la contrainte induite. La jauge PVDF apporte une contribution nouvelle et inhabituelle en métrologie des ondes de choc.

La jauge fonctionne en mode dérivatif dans le cas de nos essais expérimentaux : la charge électrique déterminée par intégration numérique en fonction du temps apparaît être l'image du profil de l'onde de choc [4]. La charge électrique est aisément corrélable à la valeur de la contrainte atteinte à l'équilibre.

Le capteur PVDF est un matériau très complexe. La phase ferroélectrique responsable de ses propriétés piézoélectriques et pyroélectriques est induite après étirage mécanique.

Les domaines ferroélectriques produits mécaniquement sont orientés, et leur alignement suivant l'axe électrique doit être contrôlé lors de la polarisation.

La polarisation du PVDF ferroélectrique [1 à 5 ] confère au capteur standard une valeur de polarisation rémanente reproductible. Les propriétés du PVDF en dépendent $[4,6]$. Le procédé ISL [5] permet de mesurer cette polarisation rémanente et de l'ajuster à la valeur nominale choisie. Celle-ci est égale à $9,2 \mu \mathrm{C} / \mathrm{cm}^{2} \pm 0,2 \mu \mathrm{C} / \mathrm{cm}^{2}$ pour les jauges fabriquées par METRAVIB. II possède l'avantage considérable de drainer les ions et les charges d'espace à l'intérieur du matériau. II assure une parfaite homogénéité de la distribution spatiale en volume de la polarisation [6]. En effet, seul le respect de la condition $\operatorname{div} \vec{D}=0(\vec{D}$ : vecteur induction électrique) conduit à un capteur possédant une réponse piézoélectrique reproductible $[6,7]$.

La configuration des jauges ISL METRAVIB a déjà été décrite [10].

D'autres jauges PVDF faisant appel à une nouvelle technologie développée à I'ISL, et polarisées de façon précise à des niveaux compris entre 6 et $9 \mu \mathrm{C} / \mathrm{cm}^{2}$, ont été réalisées à l'ISL. Elles possèdent en plus la particularité de présenter une très faible inductance.

Chaque échantillon utilisé a sa propre histoire électrique et l'on connaît sa polarisation rémanente déterminée par la mesure du cycle d’hystérésis [5].

\section{3 - Résultats}

\section{A - Réponse en courant du PVDF ISL METRAVIB}

Lorsque le temps de montée du courant électrique est inférieur au temps de transit de l'onde de choc dans le PVDF (c'est-à-dire au travers de son épaisseur), la valeur du premier maximum du courant libéré est corrélable à la pression de choc induite dans le PVDF. Rappelons que le courant I(t) délivré par un élément piézoélectrique d'épaisseur I soumis à une onde de choc plane est proportionnel à la différence des contraintes sur la face d'entrée et de sortie de l'élément [9].

On peut donc représenter (fig. 1) la densité de courant électrique par unité de surface en fonction 
de la pression de choc induite dans le capteur PVDF. On constate que l'allure de cette courbe est celle d'une application linéaire.

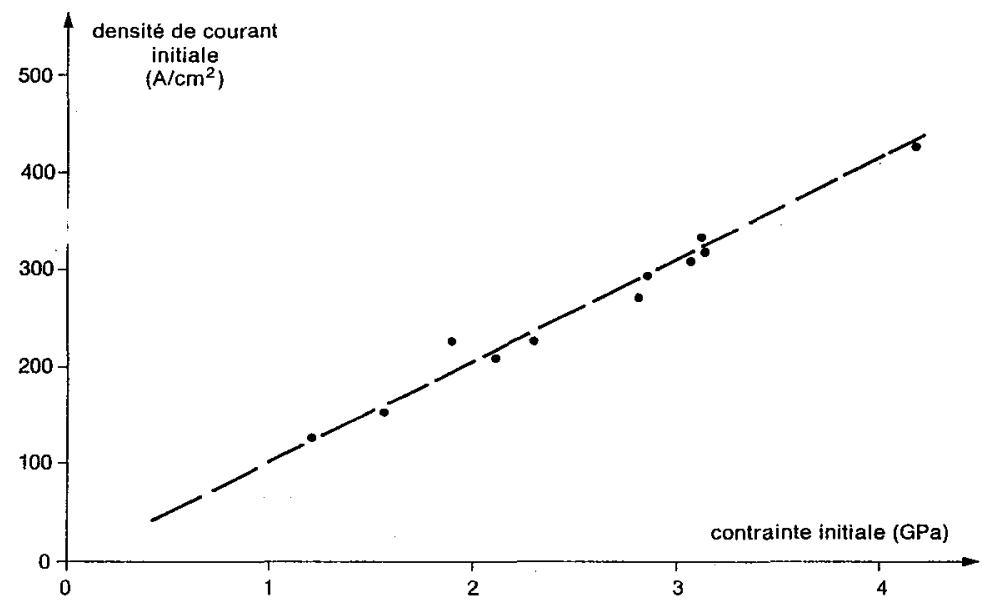

Fig. 1 : Densité de courant initiale en fonction de la contrainte initiale

\section{B - Réponse en mode "charge électrique" du PVDF ISL METRAVIB}

Nous avons vu que la jauge PVDF fonctionne en mode dérivatif et que la charge électrique apparaît être l'image du profil de l'onde de choc.

Pour les différentes pressions de choc atteintes (jusqu'à $20 \mathrm{GPa}$ ), les résultats donnant la valeur correspondante de la charge électrique figurent dans le tableau ci-dessous.

\begin{tabular}{|c|c|c|c|c|c|}
\hline Impacteur/cible & $\begin{array}{l}\text { vitesse } \\
\text { d'impact } \\
\text { (km/s) }\end{array}$ & $\begin{array}{c}\text { contrainte } \\
\text { initiale } \\
(\mathrm{GPa})\end{array}$ & $\begin{array}{c}\text { contrainte } \\
\text { l'équilibre } \\
\text { (GPa) }\end{array}$ & $\begin{array}{c}\text { densité de } \\
\text { courant } \\
\left(\mathrm{A} / \mathrm{cm}^{2}\right)\end{array}$ & $\begin{array}{l}\text { charge } \\
\left(\mu \mathrm{C} / \mathrm{cm}^{2}\right)\end{array}$ \\
\hline Kel-F Kel-F & 0,598 & 1,61 & 1,61 & 27,6 & 1,522 \\
\hline Quartz Quartz & 0,269 & 1,05 & 2,33 & 79,8 & 1,796 \\
\hline Quartz Quartz & 0,325 & 1,30 & 2,86 & 150,5 & 2,080 \\
\hline Quartz Quartz & 0,402 & 1,66 & 3,53 & 230,4 & 2,440 \\
\hline Quartz Quartz & 0,536 & 2,32 & 4,78 & 271,3 & 2,742 \\
\hline Saphir Saphir & 0,271 & 1,26 & 6,15 & 126,2 & 3,243 \\
\hline Saphir Saphir & 0,426 & 2,15 & 9,73 & 209,5 & 3,727 \\
\hline Saphir Saphir & 0,462 & 2,37 & 10,57 & 226,3 & 4,047 \\
\hline Saphir Saphir & 0,523 & 2,76 & 12,00 & 295.9 & 4,202 \\
\hline Saphir Saphir & 0,563 & 3,02 & 12,94 & 309,2 & 4.445 \\
\hline Saphir Saphir & 0,573 & 3,09 & 13,18 & 334,4 & 4,374 \\
\hline Saphir Saphir & 0,576 & 3,11 & 13,25 & 320,6 & 4,367 \\
\hline Saphir Saphir & 0,703 & 4,00 & 16,26 & 442,9 & 4,604 \\
\hline Saphir Saphir & 0,708 & 4,03 & 16,38 & 423,3 & 4,880 \\
\hline NC WC & 0,410 & 2,15 & 18,81 & - & 5,349 \\
\hline WC WC & 0,428 & 2,26 & 19,55 & - & 5,429 \\
\hline WC WC & 0,479 & 2,60 & 21,64 & - & 5,457 \\
\hline
\end{tabular}

$W C=$ carbure de tungstène 
De 0 à $10 \mathrm{GPa}$ la précision sur la charge électrique est de l'ordre de $3 \%$. De 15 à 20 GPa la précision diminue mais reste de l'ordre de $6 \%$.

La réponse piézoélectrique est bien une fonction continue de la pression de choc $[8,10,11]$.

Pour essayer d'appréhender l'origine de certaines déviations observées au-dessus de $15 \mathrm{GPa}$, des jauges PVDF faisant appel à une nouvelle technologie mise au point à l'ISL et polarisées de façon très précise et possédant une faible inductance, ont été soumises aux pressions de choc suivantes : 2,5 et $21 \mathrm{GPa}$.

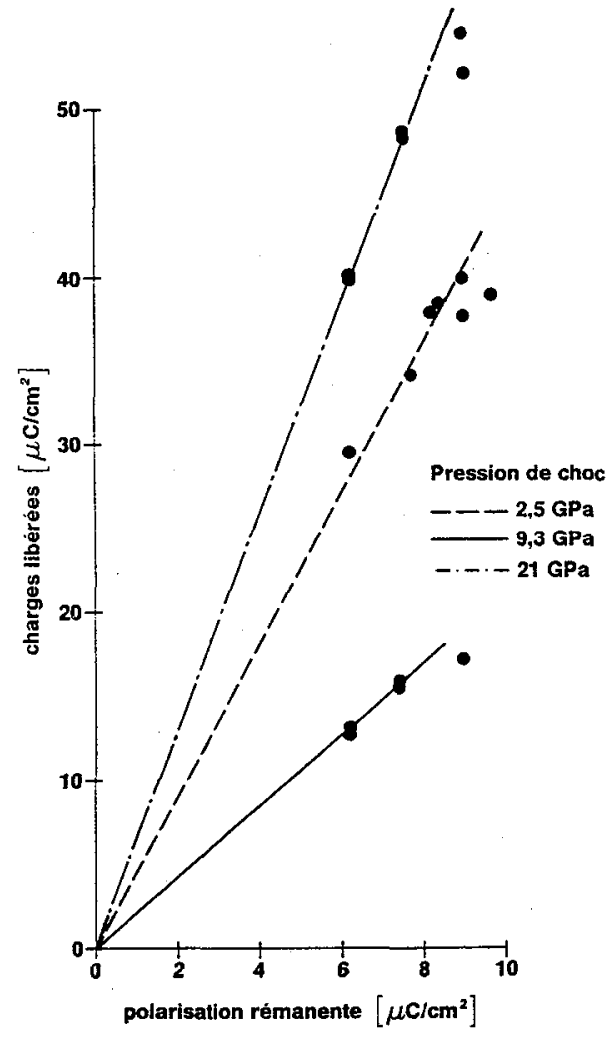

Fig. 2

La charge électrique est une fonction linéaire de la polarisation rémanente à pression de choc constante (jauges PVDF ISL) (fig. 2). Des résultats identiques obtenus par R.A. GRAHAM à une pression de 9,3 GPa sont représentés sur cette même figure. On remarque que plus la pression de choc est élevée, plus la réponse de la jauge ISL-METRAVIB s'écarte de façon aléatoire des courbes représentées en figure 2 .

Ces résultats montrent que :

- la mesure de la polarisation apparaît être imprécise à des niveaux supérieurs à $8 \mu \mathrm{C} / \mathrm{cm}^{2}$,

- la dispersion est inférieure à $3 \%$ pour les nouvelles jauges ISL,

- la réponse d'une jauge peut être directement déterminée à partir de ces caractéristiques. 


\section{4 - Conclusion}

L'étude de la réponse sous choc des jauges PVDF ISL METRAVIB a montré les qualités de ce capteur piézoélectrique dans la gamme $0,3-20 \mathrm{GPa}$, ainsi que ses légères imperfections entre 15 et $20 \mathrm{GPa}$, dont le fabricant tiendra compte.

Cette étude montre par ailleurs qu'un élément PVDF

- polarisé de façon précise,

- faiblement inductif,

répond de façon plus précise aux hautes pressions. II est un candidat de choix pour constituer un capteur performant pour la mesure des pressions de choc de forte intensité.

L'étude de tels capteurs de seconde génération fait l'objet de travaux actuels.

\section{Références}

11/ BAUER, F, Behavior of Ferroelectric Ceramics and PVDF Polymers Under Shock Loading, Proceedings of Shock Waves in Condensed Matter, 1981, W.J. Nellis, L. Seaman, R.A. Graham (Eds), American Institute of Physics (1982), p. 251

12/ BAUER, F, Piezoelectric and Electric Properties of PVDF Polymers Under Shock Wave Action: Application to Shock Transducers, Proceedings of Shock Waves in Condensed Matter, 1983, J.R. Asay, R.A. Graham, G.K. Straub, North Holland (1984), p. 225

13/ BAUER F, PVDF Polymers: Ferroelectric Polarization and Piezoelectric Properties Under Dynamic Pressure and Shock Wave Action, Ferroelectrics, Vol. 49, p. 231, 1983

14/ BAUER F, Properties and High Pressure Shock Loading Response of Poled Ferroelectric PVDF Polymer Gauges, Proceedings of Techniques and Theory of Stress Measurements for Shock Wave Applications, R.B. Stout, F.R. Norwood, M.E. Fourney (Eds), New York, ASME, pp. 19-28, 1987

15/ BAUER F, Method and Apparatur for Polarizing Ferroelectric Materials, French Patent 82221025, U.S. Patents 4611260 and 4684337

16/ ALQUIÉ, C, LEWINER, J, A New Method for Studying Piezoelectric Materials, Rev. Phys. Appliquée, Vol. 20, p. 131, 1985

17/ REED, R.P, Recent Developments in Piezoelectric Polymer Stress Gauges, Proceedings of the Range Commanders Council, Instrumentation/Transducer Committee, 14th Transducer Workshop, S.F. Kuehn (Ed.), Colorado Springs/CO, USA, June 15-18, 1987

18/ LEE L.M, WILLIAMS W.D, GRAHAM R.A, BAUER F, Studies of Bauer Piezoelectric Polymer Gauges (PVDF) Under Impact Loading, Proceedings of Shock Waves in Condensed Matter, Y.M. Gupta (Ed.), New York, Plenum Press, p. 497, 1986

/9/ GRAHAM R.A, Communication privée 
110/ LEE L.M, et al., Standardized Bauer PVDF Piezoelectric Polymer Shock Gauges, Proceedings of DYMAT, Ajaccio 1988, Les éditions de physique, France

111/ GRAHAM R.A, LEE L.M, BAUER F, Response of Bauer Piezoelectric Polymer Stress Gauges (PVDF) to Shock Loading, Proceedings of Shock Waves in Condensed Matter, Elsevier Sc. Publ. B.V., p. 619,1988

112/ LASL Shock Hugoniot Data, Stanley P. Marsh (Ed.), University of California Press, Berkeley/CA, USA

113/ BAUER F, Thèse Lyon 1977, n I-DE 77003 